\title{
Drugs based on natural compounds: recent achievements and future perspectives
}

\author{
Virginia Lanzotti
}

Published online: 31 October 2014

(C) Springer Science+Business Media Dordrecht 2014

In recent years, despite enormous efforts to develop new drugs by sophisticated approaches, there has also been a trend for rediscovery of natural products uses. In this context, new metabolites with unusual chemical structures and promising biological and pharmacological properties have inspired chemists in their synthetic approach searching for more potent and selective drugs. At the same time, the increasing awareness on prevention of common diseases such as atherosclerosis and cancer suggested an improvement of life style, including better diets by consumption of natural foods. These topics have been the focus of the Conference on Natural Products in Cancer Prevention and Therapy, 2nd Edition, a meeting hosted by the University of Naples "Federico II" on behalf of the Phytochemical Society of Europe.

As chairman of the organizing committee, I have been very honoured and pleased by the large participation of over 120 scientists from 25 countries, not limited to Europe, but including also Africa, America and Asia.

The aim of the congress was the presentation of recent results and the exchange of ideas in the field of natural products in cancer prevention and therapy, but also the discussion of new trends and general aspects of research in phytochemistry and medicinal chemistry.

V. Lanzotti $(\bowtie)$

Dipartimento di Agraria, University of Naples Federico II, Via Università 100, 80055 Portici, Naples, Italy

e-mail: lanzotti@unina.it
Higher plants continue to be the source of many new drugs used for a variety of diseases. This important matter has been discussed by Kinghorn and co-workers involved in a collaborative multi-institutional project directed towards the discovery of new antitumor agents. In their paper, opening this special issue, the isolation and characterization of new secondary metabolites from tropical plants is described together with data regarding their activity and selectivity for cancer cells. Some of these natural product lead candidates, discovered through bioactivity guided fractionation, are proposed as scaffolds for synthetic modifications to develop new potential anticancer agents.

The article by Roussis and co-workers reports on the analysis of 46 different Pinus species, affording the identification of 161 metabolites, mainly volatiles, from the pine foliage oils. The work is a representative example of traditional extensive phytochemical research consisting of a comparative metabolomic characterization of plants aimed to find new compounds of potential pharmaceutical interest.

Recent epidemiological studies reported that an increase of consumptions of Allium species reduce the risk of prostate and gastric cancers. This could be related to two main classes of compounds: the apolar sulfur compounds and the polar saponins, the latter being pungent and more stable during cooking. On this base, the third contribution by Lanzotti et al. reviewed the literature about the major volatile and non-volatile organic compounds of garlic and other Allium plants 
focusing on their antibacterial and cytotoxic activity with related mechanisms of action.

Breast cancer is one of the most frequently diagnosed cancers and major cause of death in women in the world. Bishayee and co-workers reviewed the potential role of oleanolic acid, oleanane triterpenoids and related synthetic compounds in the chemoprevention and treatment of mammary neoplasia. Both in vitro and in vivo studies on these agents and related molecular mechanisms are presented. Several challenges and future directions of research to translate already available impressive preclinical knowledge to clinical practice of breast cancer prevention and therapy are also presented.

Ahmad and co-workers reviewed data on the use of plant-based herbal medicine for complementing the current treatments as adjuvant or alternative therapy. Many studies in cell lines, animal models and human epidemiological trials suggested a protective role of a large number of molecules of herbal origin against different types of cancers. Thus, cancer control may therefore benefit from the anti-cancer potential of alternative therapies with herbal treatment whose curative action is known since ancient times.

Diederich and co-workers reviewed the cytotoxicity and anti-cancer mechanism of action of known compounds from Goniothalamus species growing in tropical and subtropical Asia intensively used for medicinal purposes. Interestingly, such compounds strongly affect the resistance to apoptotic cell death and the de-regulation of cell cycle thus constituting a promising source of potential scaffolds for further structural modifications.

Dietary polyphenolics have been proposed to play a role in cancer prevention although, at present, there is not yet a satisfactory explanation on their action mechanism. Arroo et al. addressed this problem reporting about the activity of polymethoxy flavonoids of Citrus peel and their potential interest as cytostatic products.

Colorectal cancer is one of the leading causes of cancer incidence and death worldwide second to lung cancer. Epidemiological studies demonstrated that adherence to the Mediterranean Diet is protective toward colon cancer development. Ricciardiello and co-worker addressed this field describing several bioactive compounds from olive oil, different vegetables, and fish as preventive agents.

Dekker and co-worker reviewed the literature on composition and nutritional value of Marula fruits and oil which are characterized by high content of vitamin
$\mathrm{C}$, antioxidant property, being also source of protein, oil, magnesium, phosphorus and potassium. This is discussed in terms of potential usefulness as food supply in the diet of rural communities.

The genus Linum includes many species known to contain lignans with strong cytotoxic and antiviral activities which are the starting material of various semisynthetic derivatives used in chemotherapy, such as etoposide, teniposide and etopophos. The review of Strnad and coworkers describes the in vitro production of aryltetralin lignans from various Linum species and possible biotechnological strategies to improve their production.

Multidrug resistance (MDR) is the major obstacle for cancer chemotherapy and result from several mechanisms, including an increased drug efflux, due to overexpression of P-glycoprotein (P-gp) that transports anticancer drugs out of the cells. One of the strategies proposed to reduce this effect is the identification of compounds that selectively inhibit resistant cells. This is the subject reported by Ferreira et al. about the identification of diterpenes, triterpenes and phenolics from plants and related P-gp inhibition ability on resistant cancer cells.

Traditional approaches of research of new drugs are based on random screening procedures searching for bioactive compounds from different sources. Despite the major scientific, economic and industrial efforts, the success of such methodology has been significantly limited by side effects reported by the new drugs, such as toxicity and pharmacological resistance. The review by Mazzoleni et al. reports about the use of nucleic acids as drugs for biological control. The very recent discovery of a species specific inhibitory effect of extracellular DNA on different organisms suggests a possible new scenario of pharmacological application with potential major advantages in the process of drug development.

I wish to express my thanks to all the authors that contributed to this special issue and to the colleagues who took part in the review process. Many thanks are due to the Editor in Chief, Robert Verpoorte, for inviting me to edit this issue and to the Editorial Assistants, Arun Sundar, Suganya Manikandan and Vairamuthu Ganesan, for their valuable support.

I believe that this issue, while being a good presentation of the research state of art in this field, it will also represent a basis for discussion on new ideas stimulating future work on natural products in drug discovery and development. 